\section{MS5-P32 pH-profile analysis of $\beta$-amylase/maltose complex crystal measured at room temperature}

Bunzo Mikami ${ }^{1}$, Hirokazu Kawamura ${ }^{1}$, Kimihiko Mizutani ${ }^{1}$, Nobuyuki Takahashi ${ }^{1}$

1. Laboratory of Applied Structural Biology, Graduate School of Agriculture, Kyoto University, Japan

email: mikami@kais.kyoto-u.ac.jp

$\beta$-amylase catalyzes the liberation of maltose from the non-reducing ends of $\alpha-1$, 4-glucan such as starch and glycogen. In contrast to $\alpha$-amylase, $\beta$-amylase produces $\beta$-anomeric maltose, and is classified as an inverting enzyme. Though the structural features of $\beta$-amylases have been clarified, the detailed enzymatic mechanism of $\beta$-amylase action has not been well understood especially in the hydrolytic process including the activation of glycoside linkage to be cleaved. In soybean $\beta$-amylae (SBA), the hydrolysis of the $\alpha-1$, 4-glucosyl linkage is proceeded by two catalytic residues, Glu186 (acid) and Glu380 (base). The enzyme has two mobile loops, flexible loop (residue 96-103) and inner loop (residue 341-345) near the active site. The conformation of these loops change during enzyme action. In this paper, we are intended to determine the structural changes of SBA/maltose complex in a different $\mathrm{pH}$ media. In order to control pH correctly, we have determined the crystal structure at room temperature to avoid the undesirable effect of freezing and cryo-protectant such as glycerol. SBA was crystallized by a hanging-drop vapor diffusion $(10 \mathrm{ml}$ of $10 \mathrm{mg} / \mathrm{ml}$ enzyme) against $1 \mathrm{ml}$ of the bottom solution containing $45 \%$ saturated ammonium sulfate. The obtained crystals larger than $0.3 \mathrm{~mm}$ length were packed in glass capillaries after soaked with $200 \mathrm{mM}$ maltose in the different $\mathrm{pH}$ buffer, containing $45 \%$ saturated ammonium sulfate for $30 \mathrm{~min}$ at $20^{\circ} \mathrm{C}$. More than ten diffraction data sets were collected at room temperature with a CCD detector at BL26B1 beam-line at SPring-8. The crystal of SBA belonged to a space group of $P 3$ 21.The crystal data were collected with $98-100 \%$ completeness and $\mathrm{R}_{\text {merge }}$ of $0.04-0.05$ up to $1.58-1.68 \AA$ resolution. The modefs were refined with SHELXL program including protein anisotropic B-factors. The refined models contains one molecules of SBA comprising 492 amino acid residues, 3-7 sulfate ions and 403-450 water molecules with $\mathrm{R}=0.12-0.13$ and $\mathrm{R}_{\text {free }}=$ 0.14-0.16. At pH 4.9-7.8, two maltose molecules were located at the subsites $-2 \sim-1$ and $+1 \sim+2$ with $\alpha$-anomer boat form at subsite -1 , whereas $\alpha / \beta$-anomer chair forms were clearly found at $\mathrm{pH}$ below 4.4 (Fig. 1). This indicates that the glucose residue at subsite -1 is distorted to boat form by the deprotonation of a protein residue in the active site. The elucidation of the structure significance of the distorted sugar form on the catalytic process of this enzyme is now in progress.

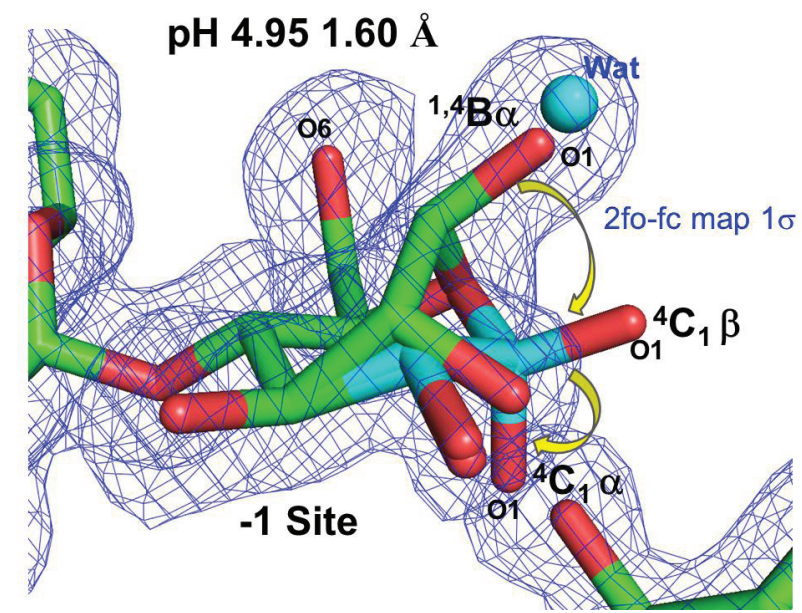

Figure 1. The effect of $\mathrm{pH}$ on the binding mode of maltose in the active site of SBA.

Keywords: beta-amylase, enzyme/substrate complex, enzyme catalytic mechanism 